\title{
Dutch cardiology residents and the COVID-19 pandemic: Every little thing counts in a crisis
}

\author{
W. R. Berger · V. Baggen · V. M. M. Vorselaars · A. C. van der Heijden · G. P. J. van Hout · G. F. L. Kapel · \\ P. Woudstra for the Junior Board (Juniorkamer) of the Netherlands Society of Cardiology (NVVC)
}

Accepted: 26 October 2020 / Published online: 3 November 2020

(C) The Author(s) 2020

\begin{abstract}
The COVID-19 pandemic has overwhelmed healthcare systems worldwide, and a large part of regular cardiology care came to a quick halt. A Dutch nationwide survey showed that $41 \%$ of cardiology residents suspended their training and worked at COVID-19 cohort units for up to 3 months. With tremendous flexibility, on-call schedules were altered and additional training was provided in order for residents to be directly available where needed most. These unprecedented times have taught them important lessons on crisis management. The momentum
\end{abstract}

\section{W. R. Berger}

Department of Cardiology, Onze Lieve Vrouwe Gasthuis, Amsterdam, The Netherlands

V. Baggen

Department of Cardiology, Erasmus Medical Centre, Rotterdam, The Netherlands

V. M. M. Vorselaars

Department of Cardiology, St. Antonius Hospital,

Nieuwegein, The Netherlands

\section{A. C. van der Heijden}

Department of Cardiology, Leiden University Medical Centre, Leiden, The Netherlands

\section{G. P. J. van Hout}

Department of Cardiology, University Medical Centre Utrecht, Utrecht, The Netherlands

\section{G. F. L. Kapel}

Department of Cardiology, Medisch Spectrum Twente, Enschede, The Netherlands

\section{P. Woudstra $(\bowtie)$}

Department of Cardiology, Heart Center, Amsterdam UMC, University of Amsterdam, Amsterdam Cardiovascular Sciences, Amsterdam, The Netherlands

Department of Cardiology, Medisch Centrum Leeuwarden, Leeuwarden, The Netherlands

p.woudstra@amsterdamumc.nl is used to incorporate novel tools for patient care. Moreover, their experience of pandemic and crisis management has provided future cardiologists with unique skills. This crisis will not be wasted; however, several challenges have to be overcome in the near future including, but not limited to, a second pandemic wave, a difficult labour market due to an economic recession, and limitations in educational opportunities.

Keywords COVID-19 · Cardiology · Training

The COVID-19 pandemic has hit cardiology patients hard, as they are susceptible to a severe course of their disease [1]. Due to the very rapid and unprecedented increase of COVID-19 patients, the regular cardiology care came to a quick halt. Cardiology residents all over the Netherlands were reallocated to COVID19 cohort units. With tremendous flexibility, on-call schedules were altered and additional training was provided in order for residents to be directly available where needed most.

A questionnaire of the Junior Board (Juniorkamer) of the Netherlands Society of Cardiology (NVVC) showed that $41 \%$ of cardiology residents were involved in frontline COVID-19 care throughout the Netherlands (Fig. 1). The questionnaire was completed by 122 residents from 36 clinics in the Netherlands; residents at every stage of the 6-year training programme from all 15 so-called 'A-clinics' (i.e. clinics which are leading the training programme) were included. They worked at COVID-19 cohort units for 1-3 months, while regular training programmes were suspended. When the pandemic hit hard, these young doctors felt a great responsibility to do whatever they could-within their competencies-and to do their 
Fig. 1 Results of nationwide survey among 122 Dutch cardiology residents during COVID-19 pandemic
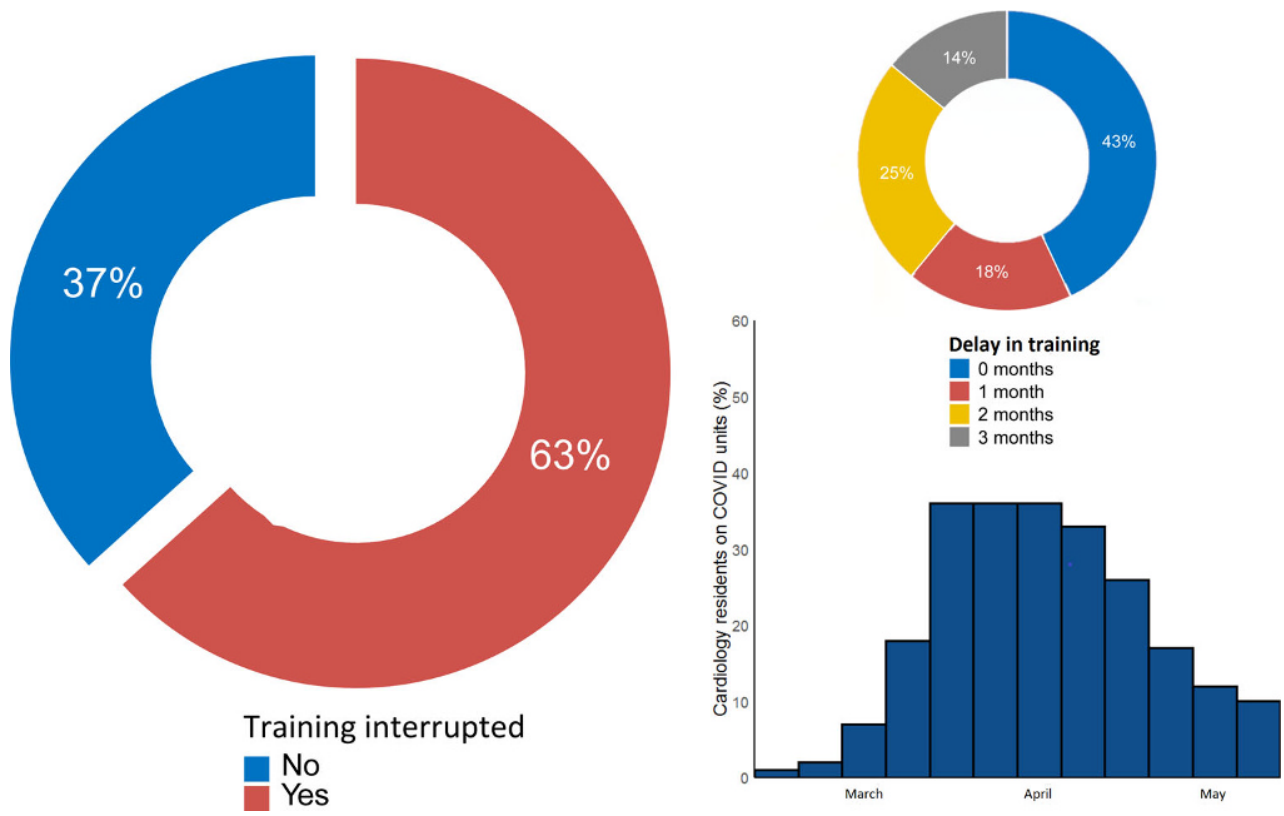

share on the wards and intensive care units. A similar pattern was seen worldwide [2].

The willingness of staff to enable reallocation of a large part of the residents proved to be of great support. After the first decline in the number of COVID19 patients in Dutch hospitals, it is time to think about the lessons learned and to reshape the future.

\section{Lessons learned}

The impact of this pandemic on regular healthcare could not have been predicted. The tremendous need of resources urged nurses, physicians and supportive staff to rethink processes of daily care in order to continue acute care, to prevent spread of the coronavirus and to limit the use of scarce protection gear. Residents were directly involved in crisis management. With their great day-to-day working experience in patient care, they helped to redefine the processes of emergency, clinical and outpatient care. While physicians are trained to be ready, the magnitude of this crisis could only be dealt with using real-life experience.

The COVID-19 crisis has shown the importance of teamwork in healthcare. Residents have shown flexibility in both the continuation of regular healthcare for the (acute) cardiac patient and dedicated care for COVID-19 patients. Moreover, the efforts of cardiologists who were involved in tasks that are normally performed by residents increased flexibility and warranted continuation of regular (acute) care. Once again, the healthcare system proves to be an efficient engine that depends on a great team effort of, but not limited to, technicians, nurses, facility services, security personnel, pharmacists and stretcher-bearers [3].
COVID-19 showed its many faces in the course of time. Residents are continuously implementing their observations in day-to-day care, together with new knowledge, which has been shared by the many publications on this topic [4]. They have followed crash courses in viral infections, epidemiology, advanced respiratory care, thrombosis, haemostasis, et cetera. Moreover, cardiology residents have proven to be essential in the often ad hoc created multidisciplinary teams of doctors given their advanced knowledge of haemodynamics and interpretation of side effects of medications (e.g. chloroquine) on cardiac conduction and function $[5,6]$.

eHealth solutions were readily made available to proof their value as an efficient alternative to face-toface contact. Daily plenary teaching moments were replaced by on-demand virtual meetings. Cardiologyspecific training, as provided by the Cardiovascular Teaching Institute (CVOI), underwent fast and rigorous innovations in online medical education. Worldwide eHealth and virtual leaning opportunities have gained an enormous momentum due to the circumstances, and we know they are here to stay $[7,8]$.

The necessary measures to prevent further spread also changed behaviour and manners in patient care. A hand on the heart or a 'low bow' has replaced the now old-fashioned handshake to welcome a patient. Family visits for admitted patients were limited to a bare minimum and communications were mainly made by phone or videophone. These included emotional and difficult conversations, such as end-of-life discussions.

The COVID-19 crisis improved our abilities as a doctor; it taught healthcare workers to be aware of their behaviour and to improve their communication skills (Tab. 1). The COVID-19 pandemic showed once again our humility toward nature and rein- 
Table 1 Lessons learned from COVID-19 healthcare crisis for cardiology residents

Knowledge
- Pandemic and disease control measures
- Development of novel disease characteristics and treatment protocols
- Respiratory care on COVID-19 cohort units and intensive care units
Management
- Crisis management structures
- Opportunities for and limitations of a healthcare system
- Multidisciplinary improvement of care
Innovation
- Implementation of eHealth solutions
- Implementation of virtual learning
Communication and collaboration
- Teamwork and compassion are cornerstones of healthcare system
- Alternative (virtual) patient and family communication
- Importance of well-organised aftercare, such as peer support

forced a skill that may sometimes be forgotten during medical training: compassion. At the same time, we experienced that everyday social interaction with colleagues is of great importance to cope emotionally with the heavy workload and the often grievous impressions this crisis has brought us.

\section{Challenges in the near future}

The experiences gained during the COVID-19 pandemic have taught the residents many lessons, even though almost half of the cardiology residents reported a delay in their cardiology training of 1-3 months (Fig. 1). To prevent gaps in training or knowledge, a personalised restructuring of the training programme is necessary for many cardiology residents. This new training scheme will be implemented in an era in which several important constraints to daily care resulting from social distancing are still valid. This could limit the exposure of residents to clinical cases and training procedures. However, we have to utilise the current circumstances to introduce new training methods, such as virtual reality education, distance learning or advanced teaching, to improve learning efficiency. Residents have to work together with their mentors in teaching hospitals and to keep being creative and flexible in order to create practical solutions.

The pandemic has an enormous economic impact, also on the Dutch healthcare system. We hope that the (financial) uncertainties that lie ahead do not hinder the future careers of cardiology residents. These future cardiologists, who conquered COVID-19 in the front- lines of healthcare with tremendous effort and flexibility, are well prepared for a great future in clinical care. The lessons they learned will be of great importance for a paradigm shift to a more pandemic-resistant society and a modern healthcare system with an accelerated introduction of eHealth solutions. Since we are in a second wave of coronavirus infections, we need to work together with all stakeholders to be prepared for the (near) future.

Conflict of interest W.R. Berger, V. Baggen, V.M.M. Vorselaars, A.C. van der Heijden, G.P.J. van Hout, G.F.L. Kapel and P. Woudstra declare that they have no competing interests.

Open Access This article is licensed under a Creative Commons Attribution 4.0 International License, which permits use, sharing, adaptation, distribution and reproduction in any medium or format, as long as you give appropriate credit to the original author(s) and the source, provide a link to the Creative Commons licence, and indicate if changes were made. The images or other third party material in this article are included in the article's Creative Commons licence, unless indicated otherwise in a credit line to the material. If material is not included in the article's Creative Commons licence and your intended use is not permitted by statutory regulation or exceeds the permitted use, you will need to obtain permission directly from the copyright holder. To view a copy of this licence, visit http://creativecommons.org/licenses/by/4.0/.

\section{References}

1. Driggin E, Madhavan MV, Bikdeli B, et al. Cardiovascular Considerations for Patients, Health Care Workers, and Health Systems During the COVID-19 Pandemic. J Am Coll Cardiol. 2020;75:2352-71.

2. Rao P, Diamond J, Korjian S, et al. The Impact of the COVID19 Pandemic on Cardiovascular Fellows-in-Training: A National Survey. JAm Coll Cardiol. 2020;76:871-5.

3. Vendrik J, de Boer J, Zwiers W, et al. Ongoing transcatheter aortic valve implantation (TAVI) practice amidst a global COVID-19 crisis: nurse-led analgesia for transfemoral TAVI. Neth HeartJ. 2020;28:384-6.

4. Van den Heuvel FMA, Vos JL, Koop Y, et al. Cardiac function in relation to myocardial injuryin hospitalised patients with COVID-19. Neth Heart J. 2020;28:410-7.

5. Sinkeler FS, Berger FA, Muntinga HJ, et al. The risk of QTcinterval prolongation in COVID-19 patients treated with chloroquine. Neth Heart J.2020;28:418-23.

6. Van den Broek MPH, Möhlmann JE, Abeln BGS, et al. Chloroquine-induced QTc prolongation in COVID-19 patients. Neth Heart J. 2020;28:406-9.

7. Almarzooq ZI, Lopes M, Kochar A. Virtual Learning During the COVID-19 Pandemic: A Disruptive Technology in Graduate Medical Education. JAm Coll Cardiol. 2020;75:2635-8.

8. DeFilippis EM, Stefanescu Schmidt AC, Reza N. Adapting the Educational Environment for Cardiovascular Fellowsin-Training During the COVID-19 Pandemic. J Am Coll Cardiol. 2020;75:2630-4. 